\title{
Note on massless and partially massless spin-2 particles in a curved background via a nonsymmetric tensor
}

\author{
H. G. M. Fortes ${ }^{*}$ and D. Dalmazi ${ }^{\dagger}$ \\ UNESP_Campus de Guaratinguetá-DFQ Avenida Dr. Ariberto Pereira da Cunha, \\ 333 CEP 12516-410-Guaratinguetá-SP-Brazil
}

(Received 3 October 2018; published 28 January 2019)

\begin{abstract}
In the last few years, we have seen an increase interest on gravitational waves due to recent and striking experimental results confirming Einstein's general relativity once more. From the field theory point of view, gravity describes the propagation of self-interacting massless spin-2 particles. They can be identified with metric perturbations about a given background metric. Since the metric is a symmetric tensor, the massless spin-2 particles present in the Einstein-Hilbert (massless Fierz-Pauli) theory are naturally described by a symmetric rank-2 tensor. However, this is not the only possible consistent massless spin-2 theory at linearized level. In particular, if we add a mass term, a new one parameter $\left(a_{1}\right)$ family of models $\mathcal{L}\left(a_{1}\right)$ shows up. They consistently describe massive spin-2 particles about Einstein spaces in terms of a nonsymmetric rank-2 tensor. Here we investigate the massless version of $\mathcal{L}\left(a_{1}\right)$ in a curved background. In the case $a_{1}=-1 / 12$, we show that the massless spin-2 particles consistently propagate, at linearized level, in maximally symmetric spaces. A similar result is obtained otherwise $\left(a_{1} \neq-1 / 12\right)$ where we have a nonsymmetric scalar-tensor massless model. The case of partially massless nonsymmetric models is also investigated.
\end{abstract}

DOI: $10.1103 /$ PhysRevD.99.025013

\section{INTRODUCTION}

The recent increase in the studies on massive spin-2 particles [1,2] is partially due to the fact they can represent massive gravitons which may offer an alternative explanation for the accelerated expansion of the universe since they lead to a weaker gravitational interaction at large distances [3,4]. Notice, however, that there are very low experimental upper bounds on the graviton mass, for instance from the LIGO experiment of detection of gravitational waves one has $10^{-22} \mathrm{eV}$, see $[5,6]$.

Another motivation is the quite recent overcome of historical theoretical obstacles in the description of massive gravitons, like the vDVZ mass discontinuity $[7,8]$ and the existence of ghosts in the nonlinear theory [9]. They have been solved by the addition of fine-tuned nonlinear selfinteraction terms for the graviton [10,11]. In 2015, it was obtained from the dRGT models of [10] a linear covariant theory consistent with the description of massive gravitons

\footnotetext{
"hemily.gomes@gmail.com

†dalmazi@feg.unesp.br
}

Published by the American Physical Society under the terms of the Creative Commons Attribution 4.0 International license. Further distribution of this work must maintain attribution to the author(s) and the published article's title, journal citation, and DOI. Funded by SCOAP ${ }^{3}$. propagating on arbitrary backgrounds $[12,13]$. Thus, recovering previous perturbative results of $[14,15]$.

All those studies of massive spin-2 particles have considered the Fierz-Pauli (FP) theory [16] as their starting point. The FP description is based on a symmetric and traceful rank-2 tensor $h_{\mu \nu}=h_{\nu \mu}$ which propagates 5 degrees of freedom (d.o.f) in $D=4$. It can be seen as the metric fluctuation about a background metric $g_{\mu \nu}^{(0)}$.

In [17], another family of models $\mathcal{L}\left(a_{1}\right)$, where $a_{1}$ is an arbitrary real constant, has been suggested which describes massive spin-2 particles via a nonsymmetric rank- 2 tensor $e_{\mu \nu} \neq e_{\nu \mu}$ in flat spaces. We have [18] coupled a background gravitational field to $\mathcal{L}\left(a_{1}\right)$ by including also nonminimal terms and have looked for curved space generalizations of the tensor, vector and scalar constraints which are necessary in order to get rid of nonphysical d.o.f. We require that the coefficients of the nonmininal terms be analytic functions of $m^{2}$. Such restriction leads us to constraint the gravitational background to Einstein spaces.

Regarding the massless case, some authors consider the linearized Einstein-Hilbert theory (massless Fierz-Pauli) as the only possible description of massless spin-2 particles via a rank-2 tensor, see the earlier work [19], except eventually for the Weyl and transverse diffeomorphism (WTDiff) invariant theory; see e.g., [20]. If the massless Fierz-Pauli theory is embedded in a curved background, the required vector symmetry implies that the background 
must be of Einstein type, i.e., $R_{\mu \nu}=R g_{\mu \nu} / D$, which is the vacuum solution for Einstein equations with cosmological constant; see [21]. Neither the addition of nonminimal higher derivative terms nor allowing nonanalytic terms in the cosmological constant change this result. The case of the WTDIFF theory also requires Eintein spaces [22].

In the present work, we look for the massless version of $\mathcal{L}\left(a_{1}\right)$ coupled to a background, providing another possible description for massless spin-2 particles besides the massless FP model. We can compare our conclusions with those of [21].

Still, when we deal with curved spaces, there is a different situation which deserves a special attention. On maximally symmetric spaces, there is a specific value for the curvature constant $R$ in terms of $m^{2}$ which allows us to have a scalar gauge symmetry, even with $m \neq 0$. Consequently, we have a theory describing a massive spin2 particle with 4 d.o.f., instead of $5=2 s+1$ for $D=4$. This kind of theory has been intensively studied in massive gravity and it is called partially massless theories [23]. Thus, in the present work, we seek the partially massless theories corresponding to the $\mathcal{L}^{g}\left(a_{1}\right)$ models.

\section{SPIN-2 PARTICLES IN CURVED SPACES}

\section{A. Fierz-Pauli action}

The linear action for massive spin-2 particles propagating on a curved background $g_{\mu \nu}$ is usually described by the linearized Einstein-Hilbert action plus the Fierz-Pauli mass term and an extra term proportional to the scalar curvature $^{1}$ :

$$
\begin{aligned}
S= & \int d^{4} x \sqrt{-g}\left[-\frac{1}{2} \nabla_{\alpha} h_{\mu \nu} \nabla^{\alpha} h^{\mu \nu}+\nabla_{\alpha} h_{\mu \nu} \nabla^{\nu} h^{\mu \alpha}\right. \\
& -\nabla_{\mu} h \nabla_{\nu} h^{\mu \nu}+\frac{1}{2} \nabla_{\mu} h \nabla^{\mu} h+-\frac{1}{2} m^{2}\left(h_{\mu \nu} h^{\mu \nu}-h^{2}\right) \\
& \left.+\frac{R}{4}\left(h^{\mu \nu} h_{\mu \nu}-\frac{1}{2} h^{2}\right)\right],
\end{aligned}
$$

where $h_{\mu \nu}$ is a symmetric tensor $\left(h_{\mu \nu}=h_{\nu \mu}\right)$. The covariant derivatives are calculated with respect to a background metric $g_{\mu \nu}^{(0)}$. In the flat space, $g_{\mu \nu}^{(0)}=\eta_{\mu \nu}$, the theory (1) becomes the usual Fierz-Pauli theory whose massless version is the linearized Einstein-Hilbert model $(\sqrt{-g} R)_{h h}$ where $g_{\mu \nu}=\eta_{\mu \nu}+h_{\mu \nu}$.

In order to have consistency, it is necessary to obtain all the curved space Fierz-Pauli constraints,

$$
\begin{gathered}
\nabla^{\mu} h_{\mu \nu}=0 \\
h=0,
\end{gathered}
$$

\footnotetext{
${ }^{1}$ Throughout this work, we use $\eta_{\mu \nu}=(-,+,+,+)$.
}

which is achieved only in Einstein background spaces; see, e.g., [1] and references therein,

$$
R_{\mu \nu}=\frac{R}{4} g_{\mu \nu} .
$$

When we seek for the scalar constraint (3), the expression below comes up when we combine second derivatives $\nabla_{\mu} \nabla_{\nu} E^{\mu \nu}$ and the trace $g_{\mu \nu} E^{\mu \nu}$ of the equations of motion $E^{\mu \nu}=0$ :

$$
\left(3 m^{2}-\frac{R}{2}\right) h=0
$$

Thus, depending on the value of $\mathrm{m}^{2}$, we have models with different particle contents. Let us see the main results:

(i) As far as $m^{2} \neq R / 6$ and $m \neq 0$, besides the four constraints (2) we obtain from (5) the desired scalar constraint $h=0$. In this case, we are left with 5 propagating d.o.f. as expected for a massive spin- 2 model.

(ii) If $m^{2}=R / 6$, we loose the scalar constraint ${ }^{2}$ (3). Instead, the action has a scalar gauge symmetry

$$
\delta h_{\mu \nu}=\nabla_{\mu} \nabla_{\nu} \phi+\frac{m^{2}}{2} \phi g_{\mu \nu} .
$$

A scalar gauge symmetry removes 2 d.o.f. in contrast to a scalar constraint which removes only one. As a result, the model propagates 4 d.o.f. instead of 5 which is known as a "partially massless" theory [23] and it will be discussed in the subsection III.

(iii) If $m=0$, there is neither vector nor scalar constraint but conversely the action acquires the vector gauge symmetry

$$
\delta h_{\mu \nu}=\nabla_{\mu} \xi_{\nu}+\nabla_{\nu} \xi_{\mu}
$$

which is the linearized diffeomorphism symmetry of general relativity. We are left with 2 d.o.f., describing in fact a massless spin-2 particle. Furthermore, at $m=0$ the action (1) coincides with the linearized version of

$$
S_{\Lambda}=\int d^{4} x \sqrt{-g}(R-2 \Lambda)
$$

around a curved background $g_{\mu \nu}=g_{\mu \nu}^{(0)}+h_{\mu \nu}$ of the Einstein type with $R=4 \Lambda$ which seems to indicate that any massless spin-2 particle must be identified with the graviton [21] as we have mentioned before.

\footnotetext{
${ }^{2}$ The case $m^{2}=R / 6$ corresponds to the so-caled Higuchi bound [24].
} 


\section{B. $\mathcal{L}\left(a_{1}\right)$ models and their massless versions}

In [25], a family of second-order Lagrangians $\mathcal{L}\left(a_{1}\right)$ has been presented in the flat space and in arbitrary dimensions $D \geq 3$, but here we focus in $D=4$. It describes massive spin-2 particles via a nonsymmetric rank-2 tensor $e_{\mu \nu} \neq e_{\nu \mu}$. In [18], the $\mathcal{L}\left(a_{1}\right)$ models have been coupled to a curved background. In order to find massive theories on curved spaces, we minimally couple the corresponding flat space action and then add curvature terms in such a way to obtain the necessary constraints and achieve the correct number of d.o.f.,

$$
\begin{aligned}
\mathcal{L}^{g}\left(a_{1}\right)= & -\frac{1}{4} \nabla^{\mu} e^{\alpha \beta} \nabla_{\mu} e_{\alpha \beta}-\frac{1}{4} \nabla^{\mu} e^{\alpha \beta} \nabla_{\mu} e_{\beta \alpha}+a_{1} \nabla^{\alpha} e_{\alpha \beta} \nabla_{\mu} e^{\mu \beta}+\frac{1}{2} \nabla^{\alpha} e_{\alpha \beta} \nabla_{\mu} e^{\beta \mu}+\frac{1}{4} \nabla^{\alpha} e_{\beta \alpha} \nabla_{\mu} e^{\beta \mu}+\left(a_{1}+\frac{1}{4}\right) \nabla^{\mu} e \nabla_{\mu} e \\
& -\left(a_{1}+\frac{1}{4}\right) \nabla^{\mu} e \nabla^{\alpha} e_{\alpha \mu}+-\left(a_{1}+\frac{1}{4}\right) \nabla^{\mu} e \nabla^{\alpha} e_{\mu \alpha}+f_{1} R e^{\alpha \beta} e_{\alpha \beta}+f_{2} R e^{2}+f_{3} R_{\alpha \beta \mu \nu} e^{\alpha \mu} e^{\beta \nu}+f_{4} R_{\alpha \beta} e^{\alpha \mu} e^{\beta}{ }_{\mu} \\
& +f_{5} R_{\alpha \beta} e^{\alpha \beta} e+f_{6} R_{\alpha \beta \mu \nu} e^{\alpha \beta} e^{\mu \nu}+f_{7} R_{\alpha \beta} e^{\alpha \mu} e_{\mu}^{\beta}+f_{8} R e^{\alpha \beta} e_{\beta \alpha}+f_{9} R_{\alpha \beta} e^{\mu \alpha} e_{\mu}{ }^{\beta}-\frac{m^{2}}{2}\left(e_{\mu \nu} e^{\nu \mu}-e^{2}\right)
\end{aligned}
$$

The constant $a_{1}$ is a real number and $e=g^{\mu \nu} e_{\mu \nu}$. The coefficients $f_{j}, j=1 \ldots 9$, are partially fixed [18] by requiring that the curved space FP constraints are satisfied:

$$
\nabla^{\mu} e_{\mu \nu}=0=e_{[\mu, \nu]}=0=g^{\mu \nu} e_{\mu \nu}
$$

In the flat space, we recover the theory $\mathcal{L}\left(a_{1}\right)$ of [17] which describes massive spin-2 particles and whose massless part is invariant under

$$
\delta e_{\mu \nu}=\partial_{\nu} \xi_{\mu}+\partial^{\alpha} \Lambda_{[\alpha \mu \nu]},
$$

with $\Lambda_{[\alpha \mu \nu]}$ a fully antisymmetric tensor. It is interesting to split the discussion into three cases. At $a_{1}=1 / 4$ we recover the FP model, since the antisymmetric components $\left(e_{\mu \nu}-e_{\nu \mu}\right) / 2$ decouple due to the enlargement of the massless symmetries (11) by antisymmetric shifts $\delta e_{\mu \nu}=\Lambda_{\mu \nu}=-\Lambda_{\nu \mu}$. At $a_{1}=-1 / 12$ the massless symmetries (11) are augmented by Weyl transformations $\delta e_{\mu \nu}=\eta_{\mu \nu} \phi$. Finally, at $a_{1} \neq 1 / 4$ and $a_{1} \neq-1 / 12$, the particle content of $\mathcal{L}^{m=0}\left(a_{1}\right)$ consists of massless spin-2 particles plus massless spin-0 particles. The massless spin-0 particle is physical if $a_{1}>\frac{1}{4}$ or $a_{1}<-\frac{1}{12}$ and disappears at $a_{1} \neq 1 / 4$ or $a_{1} \neq-1 / 12$ whereas the spin-2 particle is always physical.

Regarding massless theories on curved spaces, we now require invariance under gauge symmetries. As in the flat case we have three cases: $a_{1}=1 / 4, a_{1}=-1 / 12$ and $a_{1} \neq 1 / 4,-1 / 12$. Since we recover the known FP theory at $a_{1}=1 / 4$, we start with $a_{1}=-1 / 12$ where we slightly change the notation from $f_{j}$ to $d_{j}$. Due to the Weyl symmetry in the massless sector there is no need anymore of the FP fine tuning of the mass term and the model is called a non-Fierz-Pauli one with any arbitrary constant $c$ in the mass term; see (12).

\section{1. $\mathcal{L}_{\mathrm{nFP}}(c)$ model $\left(a_{1}=-1 / 12\right)$}

The generalization of the massive theory $\mathcal{L}_{\mathrm{nFP}}(c)$ to curved spaces was first suggested in [18] and the main results are summarized in the Eqs. (12)-(15) below. The most general Lagrangian coupled to an Einstein background and quadratic in derivatives is given by

$$
\begin{aligned}
\mathcal{L}_{\mathrm{nFP}}^{g}(c)= & \sqrt{-g}\left[-\frac{1}{4} \nabla^{\mu} e^{\alpha \beta} \nabla_{\mu} e_{\alpha \beta}-\frac{1}{4} \nabla^{\mu} e^{\alpha \beta} \nabla_{\mu} e_{\beta \alpha}-\frac{1}{12} \nabla^{\alpha} e_{\alpha \beta} \nabla_{\lambda} e^{\lambda \beta}+\frac{1}{2} \nabla^{\alpha} e_{\alpha \beta} \nabla_{\lambda} e^{\beta \lambda}+\frac{1}{4} \nabla^{\alpha} e_{\beta \alpha} \nabla_{\lambda} e^{\beta \lambda}+\frac{1}{6} \nabla^{\mu} \nabla_{\mu} e\right. \\
& -\frac{1}{3} \nabla^{\alpha} e_{\alpha \beta} \nabla^{\beta} e-\frac{m^{2}}{2}\left(e_{\alpha \beta} e^{\beta \alpha}+c e^{2}\right)+d_{1} R^{\alpha \beta} e_{\alpha \beta}+d_{2} R e^{2}+d_{3} R_{\alpha \beta \mu \nu} e^{\alpha \mu} e^{\beta \nu}+d_{4} R_{\alpha \beta} e^{\alpha \mu} e^{\beta}{ }_{\mu}+d_{5} R_{\alpha \beta} e^{\alpha \beta} e \\
& \left.+d_{6} R_{\alpha \beta \mu \nu} e^{\alpha \beta} e^{\mu \nu}+d_{7} R_{\alpha \beta} e^{\alpha \mu} e_{\mu}^{\beta}+d_{8} R^{\alpha \beta} e_{\beta \alpha}+d_{9} R_{\alpha \beta} e^{\mu \alpha} e_{\mu}^{\beta}\right]
\end{aligned}
$$

where $d_{j}$ 's are free parameters a priori. It is necessary that the model presents the correct number of d.o.f. If we require that $d_{j}$ are all analytic functions of $m^{2}$, in order to satisfy the FP constraints (10) it is necessary [21] to restrict the background to Einstein spaces (4) and fix three parameters, 


$$
d_{1}+\frac{d_{4}}{4}+\frac{d_{9}}{4}=0, \quad d_{3}=1, \quad d_{6}=-\frac{1}{2}
$$

The equations of motion become $\mathrm{e}^{3}$

$$
E_{\rho \sigma}=\left(\square-\tilde{m}^{2}\right) e_{\rho \sigma}+2 R_{\rho \alpha \sigma \beta} e^{\alpha \beta}=0
$$

where

$$
\tilde{m}^{2} \equiv m^{2}-\left(2 d_{8}+\frac{d_{7}}{2}\right) R .
$$

Let us now consider the massless version of (12). The gauge symmetries of the flat case are given in (11) plus Weyl transformations. Now we expect

$$
\delta e_{\mu \nu}=g_{\mu \nu} \phi+\nabla_{\nu} \xi_{\mu}+\nabla^{\alpha} \Lambda_{[\alpha \mu \nu]} .
$$

By calculating the variation of the action $S_{\mathrm{nFP}}^{g, m=0}$ under (16), we obtain (under the integral):

$$
\begin{aligned}
\delta \mathcal{L}_{\mathrm{nFP}}^{g, m=0}= & \sqrt{-g}\left\{\phi\left[\left(2 d_{1}+8 d_{2}+d_{5}+2 d_{8}\right) R e+2\left(d_{3}+d_{4}+2 d_{5}+d_{7}+d_{9}\right) R_{\rho \sigma} e^{\rho \sigma}\right]+\nabla_{\nu} \xi_{\mu}\left[\left(\frac{1}{6}+d_{7}\right) R^{\mu}{ }_{\alpha} e^{\nu \alpha}\right.\right. \\
& +\left(\frac{1}{2}+d_{7}\right) R_{\alpha}^{\nu} e^{\alpha \mu}+2\left(-1+d_{3}\right) R^{\mu \beta \nu \rho} e_{\beta \rho}+\left(1+2 d_{6}\right) R^{\alpha \beta \mu \nu} e_{\alpha \beta}+\left(-\frac{1}{2}+2 d_{4}\right) R_{\beta}^{\mu} e^{\beta \nu}+\left(\frac{1}{2}+2 d_{9}\right) R^{\nu}{ }_{\beta} e^{\mu \beta} \\
& \left.+\left(\frac{1}{3}+d_{5}\right) R^{\nu \mu} e+2 d_{1} R e^{\mu \nu}+2 d_{8} R e^{\nu \mu}\right]+\nabla^{\mu} \xi_{\mu}\left[2 d_{2} R e+d_{5} R^{\alpha \beta} e_{\alpha \beta}\right]+\xi_{\mu}\left[-\frac{1}{3} e_{\lambda \nu} \nabla^{\lambda} R^{\mu \nu}-e_{\lambda \nu} \nabla^{\nu} R^{\mu \lambda}\right. \\
& \left.\left.+e_{\alpha \lambda} \nabla^{\mu} R^{\lambda \alpha}+\frac{1}{6} e \nabla_{\mu} R\right]+\left[2 R\left(d_{1}-d_{8}\right) e^{\mu \nu}+\left(2 d_{4}-d_{7}\right) R^{\mu}{ }_{\beta} e^{\beta \nu}+\left(2 d_{9}-d_{7}\right) R_{\beta}^{\nu} e^{\mu \beta}\right] \nabla^{\alpha} \Lambda_{[\alpha \mu \nu]}\right\} .
\end{aligned}
$$

We have not been able to get $\delta S_{\mathrm{nFP}}^{g, m=0}=0$ by choosing the coefficients $d_{j}$ 's. This can be noticed if we look specifically at the coefficients $\left(\frac{1}{6}+d_{7}\right)$ and $\left(\frac{1}{2}+d_{7}\right)$ which can not be canceled simultaneously. That is why we are going to restrict the background to the Einstein spaces similarly to what happened in the massive case [18].

Therefore, let us reconsider the $\mathcal{L}_{\mathrm{nFP}}^{g, m=0}$ model coupled to Einstein spaces (4). Now we have five free parameters,

$$
\begin{aligned}
\mathcal{L}^{g, m=0}= & -\frac{1}{4} \nabla^{\mu} e^{\alpha \beta} \nabla_{\mu} e_{\alpha \beta}-\frac{1}{4} \nabla^{\mu} e^{\alpha \beta} \nabla_{\mu} e_{\beta \alpha}-\frac{1}{12} \nabla^{\alpha} e_{\alpha \beta} \nabla_{\lambda} e^{\lambda \beta}+\frac{1}{2} \nabla^{\alpha} e_{\alpha \beta} \nabla_{\lambda} e^{\beta \lambda}+\frac{1}{4} \nabla^{\alpha} e_{\beta \alpha} \nabla_{\lambda} e^{\beta \lambda}+\frac{1}{6} \nabla^{\mu} \nabla_{\mu} e-\frac{1}{3} \nabla^{\alpha} e_{\alpha \beta} \nabla^{\beta} e \\
& +\tilde{d}_{1} R e^{\alpha \beta} e_{\alpha \beta}+\tilde{d}_{2} R e^{2}+d_{3} R_{\alpha \beta \mu \nu} e^{\alpha \mu} e^{\beta \nu}+d_{6} R_{\alpha \beta \mu \nu} e^{\alpha \beta} e^{\mu \nu}+\tilde{d}_{8} R e^{\alpha \beta} e_{\beta \alpha},
\end{aligned}
$$

where we have defined

$$
\begin{gathered}
\tilde{d}_{1} \doteq d_{1}+\frac{d_{4}}{4}+\frac{d_{9}}{4} \\
\tilde{d}_{2} \doteq d_{2}+\frac{d_{5}}{4} \\
\tilde{d}_{8} \doteq d_{8}+\frac{d_{7}}{4}
\end{gathered}
$$

Under the integral, we have

$$
\begin{aligned}
\delta \mathcal{L}_{\mathrm{nFP}}^{g, m=0}= & \sqrt{-g}\left\{\phi\left(2 \tilde{d}_{1}+8 \tilde{d}_{2}+\frac{d_{3}}{2}+2 \tilde{d}_{8}\right) R e+\left(\frac{1}{12}+2 \tilde{d}_{2}\right) R e \nabla^{\mu} \xi_{\mu}+\left[\left(-1+2 d_{3}+2 d_{6}\right) R^{\mu \beta \nu \alpha} e_{\beta \alpha}\right.\right. \\
& \left.\left.-\left(1+2 d_{6}\right) R^{\alpha \nu \beta \mu} e_{\alpha \beta}+\left(\frac{1}{6}+2 \tilde{d}_{8}\right) R e^{\nu \mu}+2 \tilde{d}_{1} \operatorname{Re}^{\mu \nu}\right] \nabla_{\nu} \xi_{\mu}+\left[\left(d_{3}+2 d_{6}\right) R^{\mu \beta \nu \lambda} e_{\beta \lambda}+\left(2 \tilde{d}_{1}-2 \tilde{d}_{8}\right) R e^{\mu \nu}\right] \nabla^{\alpha} \Lambda_{[\alpha \mu \nu]}\right\}
\end{aligned}
$$

\footnotetext{
${ }^{3}$ Notice that the equations of motion are not exactly the Klein-Gordon ones since they present an additional term with the Riemann curvature. By considering that the transverse condition must be satisfied, i.e., $\nabla^{\mu} h_{\mu \nu}=0$, the presence of such term is required. Otherwise, there would be an inconsistency in the calculation of the commutator $\left[\nabla^{\mu}, \square-m^{2}\right] h_{\mu \nu}$, which is non-null.
} 
Therefore, in order to have Weyl invariance, we need

$$
2 \tilde{d}_{1}+8 \tilde{d}_{2}+\frac{d_{3}}{2}+2 \tilde{d}_{8}=0 .
$$

On the other hand, invariance under the vector transformation $\delta e_{\mu \nu}^{(2)}=\nabla_{\nu} \xi_{\mu}$ needs

$$
\begin{gathered}
-1+2 d_{3}+2 d_{6}=0 \\
1+2 d_{6}=0 \\
\frac{1}{6}+2 \tilde{d}_{8}=0 \\
\tilde{d}_{1}=0 \\
\frac{1}{12}+2 \tilde{d}_{2}=0
\end{gathered}
$$

Finally, in order to get invariance under $\delta e_{\mu \nu}^{(3)}=\nabla^{\alpha} \Lambda_{[\alpha \mu \nu]}$, we demand

$$
\begin{aligned}
& d_{3}+2 d_{6}=0 \\
& \tilde{d}_{1}-\tilde{d}_{8}=0 .
\end{aligned}
$$

We see from Eqs. (26), (27), and (30) that there is no solution which makes the Lagrangian invariant under the transformations $\delta e_{\mu \nu}^{(2)}=\nabla_{\nu} \xi_{\mu}$ and $\delta e_{\mu \nu}^{(3)}=\nabla^{\alpha} \Lambda_{[\alpha \mu \nu]}$ simultaneously. Therefore, it is not possible to obtain in this case a consistent model for massless spin-2 particles propagating even on Einstein spaces. From this point of view, regarding the massless case, the model with a symmetric field $h_{\mu \nu}=h_{\nu \mu}$ given in (1) is more flexible than $\mathcal{L}_{\mathrm{nFP}}^{g}(c)$. Still, we can identify two cases with partial symmetries:

(i) Scalar and vector symmetries

It is possible to find a unique solution for the reduced system of Eqs. (23)-(28):

$$
\begin{aligned}
& \tilde{d}_{1}=0, \quad \tilde{d}_{2}=-\frac{1}{24}, \quad d_{3}=1, \\
& d_{6}=-\frac{1}{2}, \quad \tilde{d}_{8}=-\frac{1}{12}
\end{aligned}
$$

In this case, we have a model invariant under the following gauge transformation:

$$
\delta e_{\mu \nu}=g_{\mu \nu} \phi+\nabla_{\nu} \xi_{\mu} .
$$

(ii) Scalar and tensor symmetries

Analogously, from (23), (29), and (30), we have a model invariant under the gauge transformation below,

$$
\delta e_{\mu \nu}=g_{\mu \nu} \phi+\nabla^{\alpha} \Lambda_{[\alpha \mu \nu]},
$$

where we need

$$
\tilde{d}_{1}=\tilde{d}_{8}, \quad d_{3}=-2 d_{6}, \quad \tilde{d}_{2}=\frac{d_{6}}{8}-\frac{\tilde{d}_{8}}{2} .
$$

Now, we consider the model $\mathcal{L}_{\text {nFP }}^{g, m=0}$ coupled to maximally symmetric (MS) spaces which are spaces whose Riemmann tensor is given by

$$
R_{\alpha \beta \rho \sigma}=\frac{R}{12}\left(g_{\alpha \rho} g_{\beta \sigma}-g_{\alpha \sigma} g_{\beta \rho}\right) .
$$

The variation of the Lagrangian (22) can be rewritten as

$$
\begin{aligned}
\delta \mathcal{L}_{\mathrm{nFP}}^{\mathrm{MS}, m=0}= & \sqrt{-g} R\left\{\phi\left(2 \tilde{d}_{1}+8 \tilde{d}_{2}+\frac{d_{3}}{2}+2 \tilde{d}_{8}\right) e\right. \\
& +\left(-\frac{1}{12}+\frac{d_{3}}{6}+2 \tilde{d}_{2}\right) e \nabla^{\mu} \xi_{\mu} \\
& +\left(\frac{1}{4}-\frac{d_{3}}{6}-\frac{d_{6}}{6}+2 \tilde{d}_{8}\right) e^{\nu \mu} \nabla_{\nu} \xi_{\mu} \\
& +\left(\frac{1}{12}+\frac{d_{6}}{6}+2 \tilde{d}_{1}\right) e^{\mu \nu} \nabla_{\nu} \xi_{\mu} \\
& \left.+\left(\frac{d_{3}}{12}+\frac{d_{6}}{6}+2 \tilde{d}_{1}-2 \tilde{d}_{8}\right) e^{\mu \nu} \nabla^{\alpha} \Lambda_{[\alpha \mu \nu]}\right\} .
\end{aligned}
$$

In order to get $\delta \mathcal{L}_{\mathrm{nFP}}^{\mathrm{MS}, m=0}=0$, each coefficient of the expression above must be null, which means that we need to solve the system below:

$$
\begin{gathered}
2 \tilde{d}_{1}+8 \tilde{d}_{2}+\frac{d_{3}}{2}+2 \tilde{d}_{8}=0 \\
-\frac{1}{12}+2 \tilde{d}_{2}+\frac{d_{3}}{6}=0 \\
\frac{1}{4}-\frac{d_{3}}{6}-\frac{d_{6}}{6}+2 \tilde{d}_{8}=0 \\
\frac{1}{12}+2 \tilde{d}_{1}+\frac{d_{6}}{6}=0 \\
2 \tilde{d}_{1}+\frac{d_{3}}{12}+\frac{d_{6}}{6}-2 \tilde{d}_{8}=0
\end{gathered}
$$

and the solution found is

$$
\begin{array}{ll}
\tilde{d}_{1}=-\frac{1}{12}+\tilde{d}_{8}, & \tilde{d}_{2}=-\frac{1}{24}-2 \tilde{d}_{8}, \\
d_{3}=1+24 \tilde{d}_{8}, & d_{6}=\frac{1}{2}-12 \tilde{d}_{8},
\end{array}
$$


where $\tilde{d}_{8}$ is still arbitrary because only four of the five Eqs. (37)-(41) are independent. The existence of such a solution means that $\mathcal{L}_{\mathrm{nFP}}^{g, m=0}$ in maximally symmetric spaces has symmetry under the complete set of transformations (16).

When we substitute (35) and the solution (42) in (12) at $m=0$, the original Lagrangian becomes

$$
\begin{aligned}
\mathcal{L}_{\mathrm{nFP}}^{\mathrm{MS}, m=0}= & -\frac{1}{4} \nabla^{\mu} e^{\alpha \beta} \nabla_{\mu} e_{\alpha \beta}-\frac{1}{4} \nabla^{\mu} e^{\alpha \beta} \nabla_{\mu} e_{\beta \alpha} \\
& -\frac{1}{12} \nabla^{\alpha} e_{\alpha \beta} \nabla_{\lambda} e^{\lambda \beta}+\frac{1}{2} \nabla^{\alpha} e_{\alpha \beta} \nabla_{\lambda} e^{\beta \lambda} \\
& +\frac{1}{4} \nabla^{\alpha} e_{\beta \alpha} \nabla_{\lambda} e^{\beta \lambda}+\frac{1}{6} \nabla^{\mu} \nabla_{\mu} e-\frac{1}{3} \nabla^{\alpha} e_{\alpha \beta} \nabla^{\beta} e \\
& -\frac{1}{24} R^{\alpha \beta} e_{\alpha \beta}+\frac{1}{24} R e^{2}-\frac{1}{8} R e^{\alpha \beta} e_{\beta \alpha},
\end{aligned}
$$

where $\tilde{d}_{8}$ ends up being eliminated from the coefficients. Therefore, we have a unique model consistent with the description of massless spin-2 particles propagating in maximally symmetric spaces. In addition, the Lagrangian (43) describes the massless limit of the massive model $\mathcal{L}_{\mathrm{nFP}}^{g}(c)$ given in (12) with $\tilde{d}_{2}=-1 / 24$ and $\tilde{d}_{8}=-1 / 12$. It allows us to conclude that such a subcase of the massive model $\mathcal{L}_{\mathrm{nFP}}^{g}(c)$ has a consistent massless limit, at least in maximally symmetric spaces.

\section{2. $\mathcal{L}\left(a_{1}\right)$ with $a_{1} \neq(1 / 4,-1 / 12)$}

Similarly to the previous subsection, we need the massless version of the local symmetries on curved spaces given in (11) in order to obtain a consistent massless version of $\mathcal{L}^{g}\left(a_{1}\right)$ :

$$
\delta e_{\mu \nu}=\nabla_{\nu} \xi_{\mu}+\nabla^{\alpha} \Lambda_{[\alpha \mu \nu]} .
$$

Let us start from the most general Lagrangian (9) with $m=0$, where $f_{j}$ are arbitrary constants for now. The variation leads (under the integral) to

$$
\begin{aligned}
\delta \mathcal{L}^{g, m=0}\left(a_{1}\right)= & \sqrt{-g}\left\{\nabla _ { \nu } \xi _ { \mu } \left[\left(-2 a_{1}+f_{7}\right) R_{\alpha}^{\mu} e^{\nu \alpha}+\left(\frac{1}{2}+f_{7}\right) R_{\alpha}{ }^{\nu} e^{\alpha \mu}+\left(-1+2 f_{3}+2 f_{6}\right) R^{\mu \beta \nu \rho} e_{\beta \rho}+\left(1+2 f_{6}\right) R^{\nu \alpha \beta \mu} e_{\alpha \beta}\right.\right. \\
& \left.+\left(-\frac{1}{2}+2 f_{4}\right) R^{\mu}{ }_{\beta} e^{\beta \nu}+\left(\frac{1}{2}+2 f_{9}\right) R^{\nu}{ }_{\beta} e^{\mu \beta}+\left(\frac{1}{2}+2 a_{1}+f_{5}\right) R^{\nu \mu} e+2 f_{1} R e^{\mu \nu}+2 f_{8} R e^{\nu \mu}\right] \\
& +\nabla^{\mu} \xi_{\mu}\left[2 f_{2} R e+f_{5} R^{\alpha \beta} e_{\alpha \beta}\right]+\xi_{\mu}\left[-2 a_{1} e_{\lambda \nu} \nabla_{\alpha} R^{\alpha \mu \lambda \nu}-\frac{1}{2} e_{\alpha \lambda} \nabla_{\nu} R^{\mu \alpha \nu \lambda}-\frac{1}{2} e_{\alpha \lambda} \nabla_{\nu} R^{\mu \lambda \nu \alpha}\right. \\
& \left.\left.+\left(2 a_{1}+\frac{1}{2}\right)\left(e \nabla_{\nu} R^{\mu \nu}-e_{\alpha \beta} \nabla^{\beta} R^{\mu \alpha}\right)\right]+\left[2 R\left(f_{1}-f_{8}\right) e^{\mu \nu}+\left(2 f_{4}-f_{7}\right) R_{\beta}^{\mu} e^{\beta \nu}+\left(2 f_{9}-f_{7}\right) R_{\beta}^{\nu} e^{\mu \beta}\right] \nabla^{\alpha} \Lambda_{[\alpha \mu \nu]}\right\} .
\end{aligned}
$$

Once again, we have not been able to find a solution for the $f_{j}$ in such a way that $\delta \mathcal{L}^{g, m=0}\left(a_{1}\right)=0$ on arbitrary backgrounds. Therefore, again we consider $R_{\mu \nu}=\frac{1}{4} R g_{\mu \nu}$ and rewrite the variation above:

$$
\begin{aligned}
\delta \mathcal{L}^{g, m=0}\left(a_{1}\right)= & \sqrt{-g}\left\{\nabla_{\nu} \xi_{\mu}\left[\left(-1+2 f_{3}+2 f_{6}\right) R^{\mu \beta \nu \rho} e_{\beta \rho}+\left(1+2 f_{6}\right) R^{\nu \alpha \beta \mu} e_{\alpha \beta}+2 \tilde{f}_{1} R e^{\mu \nu}+\left(\frac{1}{8}-\frac{a_{1}}{2}+2 \tilde{f}_{8}\right) R e^{\nu \mu}\right]\right. \\
& \left.+\nabla^{\mu} \xi_{\mu}\left[\frac{1}{8}+\frac{a_{1}}{2}+2 \tilde{f}_{2}\right] R e+\left[\left(f_{3}+2 f_{6}\right) R^{\mu \beta \nu \lambda} e_{\beta \lambda}+2\left(\tilde{f}_{1}-\tilde{f}_{8}\right) R e^{\mu \nu}\right] \nabla^{\alpha} \Lambda_{[\alpha \mu \nu]}\right\},
\end{aligned}
$$

where we have defined

$$
\begin{gathered}
\tilde{f}_{1}=f_{1}+\frac{f_{4}}{4}+\frac{f_{9}}{4} \\
\tilde{f}_{2}=f_{2}+\frac{f_{5}}{4} \\
\tilde{f}_{8}=f_{8}+\frac{f_{7}}{4} .
\end{gathered}
$$

In order to obtain $\delta \mathcal{L}^{g, m=0}\left(a_{1}\right)=0$, we need to find a solution of the equations below: 


$$
\begin{gathered}
-1+2 f_{3}+2 f_{6}=0 \\
1+2 f_{6}=0 \\
\tilde{f}_{1}=0 \\
\frac{1}{8}-\frac{a_{1}}{2}+2 \tilde{f}_{8}=0 \\
\frac{1}{8}+\frac{a_{1}}{2}+2 \tilde{f}_{2}=0 \\
f_{3}+2 f_{6}=0 \\
\tilde{f}_{1}-\tilde{f}_{8}=0 .
\end{gathered}
$$

However, the Eqs. (52), (53), and (56) lead us to the FierzPauli massless model: $a_{1}=1 / 4$. Thus, it is not possible to obtain a massless model for $\mathcal{L}^{g}\left(a_{1}\right)\left(a_{1} \neq 1 / 4\right)$ on Einstein spaces symmetric under (44).

On the other hand, we have models with vector and tensor symmetries separately:

(i) Vector symmetry

From the Eqs. (50)-(54), we have the following solution:

$$
\begin{aligned}
& \tilde{f}_{1}=0, \quad f_{6}=-\frac{1}{2}, \quad f_{3}=1, \\
& \tilde{f}_{8}=\frac{1}{4}\left(a_{1}-\frac{1}{4}\right), \quad \tilde{f}_{2}=-\frac{1}{4}\left(a_{1}+\frac{1}{4}\right)
\end{aligned}
$$

In this specific case, the Lagrangian is invariant under the transformation $\delta^{(1)} e_{\mu \nu}=\nabla_{\nu} \xi_{\mu}$.

(ii) Tensor symmetry

Similarly, if we choose the parameters in such way that the Eqs. (55) and (56) are satisfied, i.e.,

$$
f_{3}=-2 f_{6}, \quad \tilde{f}_{1}=\tilde{f}_{8},
$$

the Lagrangian becomes invariant under $\delta e_{\mu \nu}=$ $\nabla^{\alpha} \Lambda_{[\alpha \mu \nu]}$.

Now, considering maximally symmetric spaces, the variation of the Lagrangian $\mathcal{L}^{g, m=0}\left(a_{1}\right)$ can be written (under integral) as

$$
\begin{aligned}
\delta \mathcal{L}^{\mathrm{MS}, m=0}\left(a_{1}\right)= & \sqrt{-g} R\left\{\left(-\frac{1}{24}+\frac{a_{1}}{2}+\frac{f_{3}}{6}+2 \tilde{f}_{2}\right) e \nabla^{\mu} \xi_{\mu}\right. \\
& +\left(\frac{5}{24}-\frac{a_{1}}{2}-\frac{f_{3}}{6}-\frac{f_{6}}{6}+2 \tilde{f}_{8}\right) e^{\nu \mu} \nabla_{\nu} \xi_{\mu} \\
& +\left(\frac{1}{12}+\frac{f_{6}}{6}+2 \tilde{f}_{1}\right) e^{\mu \nu} \nabla_{\nu} \xi_{\mu} \\
& \left.+\left(\frac{f_{3}}{12}+\frac{f_{6}}{6}+2 \tilde{f}_{1}-2 \tilde{f}_{8}\right) e^{\mu \nu} \nabla^{\alpha} \Lambda_{[\alpha \mu \nu]}\right\} .
\end{aligned}
$$

In order to obtain $\delta \mathcal{L}^{\mathrm{MS}, m=0}\left(a_{1}\right)=0$, we need to solve the equations below:

$$
\begin{gathered}
-\frac{1}{24}+\frac{a_{1}}{2}+\frac{f_{3}}{6}+2 \tilde{f}_{2}=0 \\
\frac{5}{24}-\frac{a_{1}}{2}-\frac{f_{3}}{6}-\frac{f_{6}}{6}+2 \tilde{f}_{8}=0 \\
\frac{1}{12}+\frac{f_{6}}{6}+2 \tilde{f}_{1}=0 \\
\frac{f_{3}}{12}+\frac{f_{6}}{6}+2 \tilde{f}_{1}-2 \tilde{f}_{8}=0
\end{gathered}
$$

for which we have found the solution

$\tilde{f}_{1}=-\frac{1}{16}+\frac{a_{1}}{4}+\tilde{f}_{8}, \quad \tilde{f}_{2}=-\frac{1}{16}-\frac{a_{1}}{4}-2 \tilde{f}_{8}$,

$f_{3}=1+24 \tilde{f}_{8}, \quad f_{6}=\frac{1}{4}-3 a_{1}-12 \tilde{f_{8}}$,

where $\tilde{f}_{8}$ is still arbitrary. The existence of such a solution means that the Lagrangian $\mathcal{L}^{g, m=0}\left(a_{1}\right)$ in maximally symmetric spaces is symmetric under the full transformation given in (44).

By replacing the solution (64) back in $\mathcal{L}^{g, m=0}\left(a_{1}\right)$ together with the fact that the space is maximally symmetric, we reach the theory below:

$$
\begin{aligned}
\mathcal{L}^{\mathrm{MS}, m=0}\left(a_{1}\right)= & -\frac{1}{4} \nabla^{\mu} e^{\alpha \beta} \nabla_{\mu} e_{\alpha \beta}-\frac{1}{4} \nabla^{\mu} e^{\alpha \beta} \nabla_{\mu} e_{\beta \alpha} \\
& -\frac{1}{12} \nabla^{\alpha} e_{\alpha \beta} \nabla_{\lambda} e^{\lambda \beta}+\frac{1}{2} \nabla^{\alpha} e_{\alpha \beta} \nabla_{\lambda} e^{\beta \lambda} \\
& +\frac{1}{4} \nabla^{\alpha} e_{\beta \alpha} \nabla_{\lambda} e^{\beta \lambda}+\frac{1}{6} \nabla^{\mu} \nabla_{\mu} e-\frac{1}{3} \nabla^{\alpha} e_{\alpha \beta} \nabla^{\beta} e \\
& -\frac{1}{24} \operatorname{Re}^{\alpha \beta} e_{\alpha \beta}+\left(\frac{1}{48}-\frac{a_{1}}{4}\right) R e^{2} \\
& +\left(-\frac{5}{48}+\frac{a_{1}}{4}\right) R e^{\alpha \beta} e_{\beta \alpha},
\end{aligned}
$$

where the parameter $\tilde{f}_{8}$ has been naturally eliminated from the coefficients again. Therefore, we have a model consistent with the description of massless spin-2 particles plus massless spin-0 particles propagating in maximally symmetric spaces. Additionally we notice that (65) is also consistent with the massless limit of the massive $\mathcal{L}^{g}\left(a_{1}\right)$ model obtained in [18] for maximally symmetric spaces with $\tilde{f}_{2}=-\left(a_{1}+1 / 4\right) / 4$.

\section{PARTIALLY MASSLESS THEORIES}

In flat spaces, the particles are classified in massive or massless. On the other hand, on curved spaces (more speciffically, in maximally symmetric spaces) there is another possible case where spin-2 particles can propagate 
a number of d.o.f. different from both massless and massive cases. The so-called partially massless theories which describe this kind of particle $[22,24,26]$ present a peculiarity: although the mass is non-null, the theory has a scalar gauge invariance which is responsible for removing one of the d.o.f. from the massive graviton. Let us see how this happens in the Fierz-Pauli theory.

In subsection II A, we have seen that in order to obtain the scalar constraint $h=0$ in the Fierz-Pauli model, we need to demand the coefficient in (5) to be non-null. However, we have not analyzed otherwise. Thus, let us consider that the coefficient of $h$ in (5) is zero which leads us to

$$
R=6 m^{2} .
$$

There is no scalar constraint $h=0$ anymore. Conversely, the theory acquires a scalar gauge symmetry:

$$
\delta h_{\mu \nu}=\nabla_{\mu} \nabla_{\nu} \phi+\frac{m^{2}}{2} g_{\mu \nu} \phi,
$$

where $\phi$ is the gauge parameter.

On the other hand, the symmetry allows us to fix the gauge $h=0$. At this point, we have the same number of d.o.f. of a massive spin-2 particle, which corresponds to 5 in $D=4$. However, even after choosing a specific gauge, there is still a residual gauge invariance. More specifically, the theory remains invariant under a subset of transformations (67). If we perform the transformation (67) again, the equations of motion and the Fierz-Pauli constraints will remain unchanged. In order to verify it, let us first demand that the trace $h$ remains null:

$$
h^{\prime}=h+g^{\mu \nu} \delta h_{\mu \nu}=0+\left(\square+2 m^{2}\right) \phi .
$$

The new trace $h^{\prime}$ will be zero if the parameter $\alpha$ satisfies the following equation:

$$
\square \phi=-2 m^{2} \phi .
$$

If we use (66) and (69), it is possible to verify that the transverse condition $\nabla^{\mu} h_{\mu \nu}=0$ will remain true and the equations of motion will not be modified.

Therefore, the residual gauge invariance given by (67) and (69) removes one more d.o.f. from the theory. As a result, we have 4 propagating d.o.f. instead of 5 which is called a partially massless theory.

The partially massless theories have been studied at the linear level [26-29] and there has been a great effort to extend the studies to the nonlinear level, despite the obstacles which have been raised [30,31]. They are of interest for the gravitational area since the equality (66) implies a direct relation between the graviton mass and the cosmological constant $(\lambda \propto R)$. As we know, the graviton mass, if it is non-null, would be very tiny, leading to an alternative to the cosmological constant problem.

Let us see the partially massless theories associated to the $\mathcal{L}_{\mathrm{nFP}}^{g}(c)$ and $\mathcal{L}^{g}\left(a_{1}\right)$ models:

(i) $\mathcal{L}_{\mathrm{nFP}}^{g}(c)$

In [18], we have discussed in detail those massive models on curved spaces. The Lagrangian $\mathcal{L}_{\mathrm{nFP}}(c)$ in maximally symmetric spaces is the following:

$$
\begin{aligned}
\mathcal{L}_{\mathrm{nFP}}^{g}(c)= & \sqrt{-g}\left[-\frac{1}{4} \nabla^{\mu} e^{\alpha \beta} \nabla_{\mu} e_{\alpha \beta}-\frac{1}{4} \nabla^{\mu} e^{\alpha \beta} \nabla_{\mu} e_{\beta \alpha}-\frac{1}{12} \nabla^{\alpha} e_{\alpha \beta} \nabla_{\lambda} e^{\lambda \beta}+\frac{1}{2} \nabla^{\alpha} e_{\alpha \beta} \nabla_{\lambda} e^{\beta \lambda}+\frac{1}{4} \nabla^{\alpha} e_{\beta \alpha} \nabla_{\lambda} e^{\beta \lambda}+\frac{1}{6} \nabla^{\mu} \nabla_{\mu} e\right. \\
& \left.-\frac{1}{3} \nabla^{\alpha} e_{\alpha \beta} \nabla^{\beta} e-\frac{m^{2}}{2}\left(e_{\alpha \beta} e^{\beta \alpha}+c e^{2}\right)-\frac{1}{24} R^{\alpha \beta} e_{\alpha \beta}+\left(\tilde{d}_{2}+\frac{1}{12}\right) R e^{2}+\left(\tilde{d}_{8}-\frac{1}{24}\right) R e^{\alpha \beta} e_{\beta \alpha}\right],
\end{aligned}
$$

where $c, \tilde{d_{2}}$, and $\tilde{d}_{8}$ remain arbitrary. The manipulation of the equations of motion obtained from $\mathcal{L}_{\mathrm{nFP}}^{g}(c)$ leads us to the necessary constraints in order to obtain the correct number of d.o.f. for a full massive theory, namely,

$$
\begin{gathered}
e_{[\mu \nu]}=0 \\
\nabla^{\mu} e_{\mu \nu}=0 \\
e=0 .
\end{gathered}
$$

On the other hand, we have noticed that for a specific value of $R$, the theory acquires a scalar gauge symmetry. More specifically, when

$$
\left(24 \tilde{d}_{2}+1\right) R=12 m^{2} c
$$

and

$$
(24 c) \tilde{d}_{8}=24 \tilde{d}_{2}-2 c+1
$$

the scalar symmetry

$$
\delta e_{\mu \nu}=\nabla_{\mu} \nabla_{\nu} \phi+\frac{R}{1+4 c} g_{\mu \nu} \phi
$$

comes up. In this case, the constraints (71) and (72) remain true. However, the coefficient of $e$ in the scalar constraint is identically null, excluding the possibility $e=0$. 
Nevertheless, as happened in the Fierz-Pauli case, we can use the symmetry (76) in order to fix the gauge $e=0$ leading us back to 5 d.o.f., which would be the correct counting for a massive spin-2 particle. But there is still a residual gauge invariance. This can be seen if, after choosing the gauge $e=0$, we perform the transformation 76)) in the field $e_{\mu \nu}$ again. As a result, we obtain that all the equations and constraints remain unchanged if

$$
\square \phi=-\frac{4 R}{1+4 c} \phi,
$$

where $R$ is given in (74). This choice removes one more d.o.f. and, consequently, we have the so-called partially massless theory for $\mathcal{L}_{\mathrm{nFP}}(c)$ with 4 d.o.f. for a partially massless spin-2 particle.

(ii) $\mathcal{L}^{g}\left(a_{1}\right)$

Analogously, there is also a value of $R$ which gives rise to a scalar symmetry for the massive model $\mathcal{L}^{g}\left(a_{1}\right)$. More specifically, if

$$
\left(6 \tilde{f}_{8}+\frac{1}{2}\right) R=3 m^{2},
$$

the massive theory becomes invariant under the transformation

$$
\delta e_{\mu \nu}=\nabla_{\mu} \nabla_{\nu} \phi+\frac{R}{12} g_{\mu \nu} \phi .
$$

Once again, we can fix the gauge $e=0$. Even after fixing the gauge, we still have a residual gauge invariance. Thus, we can remove one more d.o.f. from the theory by choosing $\phi$ in such a way that

$$
\square \phi=-\frac{R}{3} \phi,
$$

where $R$ is given in (78).

Notice that $24 \tilde{d}_{2}+1=0$ and $6 \tilde{f}_{8}+1 / 2=0$ requires a fully massless theory $(m=0)$; see (75) and (78).

\section{CONCLUSION}

Lending continuity to the previous work [18] where we have studied the coupling of the new massive models $\mathcal{L}\left(a_{1}\right)$ to curved backgrounds, in the present work we have presented the analysis of the massless versions of those models also on curved spaces.

In order to obtain the massless version of the $\mathcal{L}\left(a_{1}\right)$ model coupled to a curved background, we have required the curved space versions of the corresponding flat space gauge symmetries. As a result, we have obtained a unique model consistent with the description of a massless spin-2 particle propagating in maximally symmetric spaces. It corresponds to the massless limit of a unique massive model from [18]. Unfortunately, it was not possible to obtain the massless theories in more general background spaces with the procedure used in our study, in contrast to the massless FP case which allows the propagation of massless spin-2 particles on Einstein spaces [21]. The key point is that instead of a ten-component field $\left(h_{\mu \nu}=h_{\nu \mu}\right)$ we have now 16-component one $\left(e_{\mu \nu} \neq e_{\nu \mu}\right)$ which requires a larger symmetry, see (16), than the linearized reparametrizations (7) in order that we end up with only two helicity modes $( \pm 2)$ in the case of $\mathcal{L}_{\mathrm{nFP}}(c)$ and an extra scalar mode in the $\mathcal{L}\left(a_{1}\right)$ case. It turns out that the tensor and vector symmetries in (16) can hardly coexist on the curved space.

Additionally, partially massless theories have been found for the models $\mathcal{L}^{g}\left(a_{1}\right)$ consistently. We have been able to find models with nonvanishing mass with scalar gauge symmetries. This fact leads us to have theories which describe, for some value of $R$, massive spin-2 particles with 4 d.o.f. instead of the $5(2 \mathrm{~s}+1)$ expected ones. Here we have gone beyond the initial studies of [18] and checked that the arising scalar symmetry allows us to fix a gauge with residual symmetries consistent with 4 d.o.f.

Finally, we are currently investigating the addition of cosmological-like terms, $\quad \Delta \mathcal{L}=\sqrt{-g}\left[\Lambda_{1} e_{\mu \nu} e^{\mu \nu}+\right.$ $\Lambda_{2} e_{\mu \nu} e^{\nu \mu}+\Lambda_{3} e^{2}$, to (9) and (12) at $m=0$, altogether with singular terms on $\Lambda_{j}$ (linear in curvatures) in the gauge transformations in order to achieve more general backgrounds.
[1] K. Hinterbichler, Theoretical aspects of massive gravity, Rev. Mod. Phys. 84, 671 (2012).

[2] C. de Rham, Massive gravity, Living Rev. Relativity 17, 7 (2014).

[3] A. G. Riess et al., Observational evidence from supernovae for an accelerating universe and a cosmological constant, Astron. J. 116, 1009 (1998).
[4] S. Perlmutter et al., Measurements of omega and lambda from 42 high redshift supernovae, Astrophys. J. 517, 565 (1999).

[5] B. P. Abbott et al. (LIGO Scientific and Virgo Collaborations), Observation of Gravitational Waves from a Binary Black Hole Merger, Phys. Rev. Lett. 116, 061102 (2016). 
[6] B. P. Abbott et al. (LIGO Scientific and Virgo Collaborations), Tests of General Relativity with GW150914, Phys. Rev. Lett. 116, 221101 (2016).

[7] H. van Dam and M. J. G. Veltman, Massive and massless Yang-Mills and gravitational fields, Nucl. Phys. B22, 397 (1970).

[8] V. I. Zakharov, Linearized gravitation theory and the graviton mass, Pis'ma Zh. Eksp. Teor. Fiz. 12, 447 (1970) [JETP Lett. 12, 312 (1970)].

[9] D. G. Boulware and S. Deser, Inconsistency of finite range gravitation, Phys. Lett. 40B, 227 (1972).

[10] C. de Rham, G. Gabadadze, and A. J. Tolley, Resummation of Massive Gravity, Phys. Rev. Lett. 106, 231101 (2011).

[11] S. Hassan and R. A. Rosen, Bimetric gravity from ghostfree massive gravity, J. High Energy Phys. 02 (2012) 126.

[12] L. Bernard, C. Deffayet, and M. von Strauss, Consistent massive graviton on arbitrary backgrounds, Phys. Rev. D 91, 104013 (2015).

[13] L. Bernard, C. Deffayet, and M. von Strauss, Massive graviton on arbitrary background: Derivation, syzygies, applications, J. Cosmol. Astropart. Phys. 06 (2015) 038.

[14] I. L. Buchbinder, V. A. Krykhtin, and V. D. Pershin, On consistent equations for massive spin-2 field coupled to gravity in string theory, Phys. Lett. B 466, 216 (1999).

[15] I. Buchbinder, D. Gitman, and V. Pershin, Causality of massive spin 2 field in external gravity, Phys. Lett. B 492, 161 (2000).

[16] M. Fierz and W. Pauli, On relativistic wave equations for particles of arbitrary spin in an electromagnetic field, Proc. R. Soc. A 173, 211 (1939).

[17] D. Dalmazi, Massive spin-2 particle from a rank-2 tensor, Phys. Rev. D 87, 125027 (2013).

[18] D. Dalmazi and H. G. M. Fortes, Nonsymmetric tensor description of massive spin-2 particles in a curved background, Phys. Rev. D 95, 065028 (2017).
[19] P. van Nieuwenhuizen, On ghost-free tensor lagrangians and linearized gravitation, Nucl. Phys. B60, 478 (1973).

[20] E. Alvarez, D. Blas, J. Garriga, and E. Verdaguer, Transverse Fierz-Pauli symmetry, Nucl. Phys. B756, 148 (2006).

[21] S. Deser and M. Henneaux, A note on spin two fields in curved backgrounds, Classical Quantum Gravity 24, 1683 (2007).

[22] J. Bonifacio, P. G. Ferreira, and K. Hinterbichler, Transverse diffeomorphism and weyl invariant massive spin 2: Linear theory, Phys. Rev. D 91, 125008 (2015).

[23] S. Deser and R. I. Nepomechie, Gauge invariance versus masslessness in de sitter spaces, Ann. Phys. (N.Y.) 154, 396 (1984).

[24] A. Higuchi, Forbidden mass range for spin-2 field theory in de sitter space-time, Nucl. Phys. B282, 397 (1987).

[25] D. Dalmazi, A. L. R. dos Santos, and E. L. Mendonça, Massive "spin-2" theories in arbitrary $D \geq 3$ dimensions, Ann. Phys. (Amsterdam) 354, 385 (2015).

[26] L. Bernard, C. Deffayet, K. Hinterbichler, and M. von Strauss, Partially massless graviton on beyond Einstein spacetimes, Phys. Rev. D 95, 124036 (2017).

[27] S. Deser and A. Waldron, Gauge invariances and phases of massive higher spins in (anti-)de sitter space, Phys. Rev. Lett. 87, 031601 (2001).

[28] S. Deser and A. Waldron, Partial masslessness of higher spins in (a)ds, Nucl. Phys. B607, 577 (2001).

[29] S. Deser and A. Waldron, Null propagation of partially massless higher spins in (a)ds and cosmological constant speculations, Phys. Lett. B 513, 137 (2001).

[30] C. de Rham, K. Hinterbichler, R. A. Rosen, and A. J. Tolley, Evidence for and obstructions to nonlinear partially massless gravity, Phys. Rev. D 88, 024003 (2013).

[31] S. Garcia-Saenz and R. A. Rosen, A non-linear extension of the spin-2 partially massless symmetry, J. High Energy Phys. 05 (2015) 042. 